\title{
Emergence and interaction of twisted flux tubes in the Sun
}

\author{
V. Archontis, A. W Hood, and C. Brady \\ School of Mathematics and Statistics, University of St. Andrews, North Haugh, St. Andrews, Fife KY16 9SS, UK \\ e-mail: vasilis@mcs.st-and.ac.uk
}

Received 4 October 2006 / Accepted 23 January 2007

\begin{abstract}
Aims. We present results from numerical simulations that study the interaction of a pair of twisted, buoyant magnetic flux tubes, which rise from the solar interior into the outer atmosphere of the Sun. The aim of our new model is to reproduce some of the dynamic solar phenomena in a self-consistent manner.

Methods. We perform non-linear simulations in 2.5D numerical experiments by solving the compressible and resistive MHD equations using a Lagrangian remap, shock capturing code (Lare2D). For some aspects of the problem, we consider the evolution of the system using both uniform and locally enhanced resistivity.

Results. The two flux tubes start to rise at the same time but from a different height below the photosphere. The leading (first) tube, which is originally located nearer to the surface, rises and eventually expands above the photosphere forming a magnetized atmosphere for the upcoming system (second tube). Current sheets, high-velocity reconnection jets, plasmoids, loop brightnenings and arcade flare-like structures are formed, for the first time in such numerical experiments, self-consistently by the emergence, expansion and the dynamical interaction between the two emerging flux systems.
\end{abstract}

Key words. Sun: magnetic fields - Sun: corona - magnetohydrodynamics (MHD)

\section{Introduction}

The emergence of buoyant flux tubes from below the photosphere, the creation of active regions and their evolution and rise into the outer atmosphere of the Sun has been the subject of strong interest recently, both analytically and numerically. In fact, the interaction of emerging flux with an overlying field or the reconnection between rising flux tubes have been used to explain observational results of X-ray bright points, flares and coronal mass ejections, based on previous theoretical models, such as the Heyvaerts et al. (1977) model, the CSHKP model and the model by Shibata et al. (1995).

Most cases of 2D and 3D simulations have dealt with either the emergence of a single flux tube or a flux sheet through the photosphere and the interaction between the rising flux system and a pre-existing ambient magnetic field (see Archontis et al. 2005; Galsgaard et al. 2005; Isobe et al. 2005, and references therein). The results of these simulations show the formation of current sheets, where intense heating occurs, the formation of hot fast outflows (jets) and the creation and ejection of plasmoids.

A few simulations use more than one flux tube or multiple flux loops which rise through a stratified atmosphere. For example, Fan et al. (1998) studied the interaction of rising twisted magnetic flux tubes in the solar convection zone via 2D numerical simulations. Linton et al. (2001) performed 3D simulations to investigate the collision and reconnection of pairs of twisted flux tubes in the solar corona. They found four different classes of interaction (bounce, merge, slingshot and tunnel) and discussed the implications of their results for models of flares and coronal mass ejections. Also, Hirose et al. (2001) simulated a quadruple magnetic source model at photospheric heights. They used 2.5D MHD simulations to study how two separate magnetic systems interact and how filament eruptions and arcade flares occur as a result of such interactions.

Mackay \& van Ballegooijen (2006a,b) studied the interaction of two initially independent bipoles through a mixture of magnetic flux transport and magnetofrictional relaxation simulations. They reported on the formation and ejection of magnetic flux ropes in the solar corona and the changing connectivity of the coronal magnetic field during the evolution of the system.

Recently, Isobe et al. (2007) studied the interaction of multiple flux loops, which are formed out of a very long flux sheet originally located below the photosphere. They reported on the formation of Ellerman bombs and surges, which are formed possibly due to the compression of the dense material accumulated in between neighbouring flux loops.

Finally, Murray \& Hood (2007) performed numerical experiments to demonstrate that the interaction of flux tubes can increase the complexity of the solar interior's magnetic field. However, the subsequent emergence of this complex field results in the atmospheric flux having a simple structural form.

In this paper, we carry out 2.5D MHD simulations of a pair of twisted flux tubes as they rise from the subphotospheric layers into the solar corona. We study their interaction and report on the phenomena produced due to the dynamical collision of the two magnetic flux systems. The paper is organised as follows: Sect. 2 presents the equations and the numerical model used in the experiments. The results are discussed in Sect. 3. First, we describe the initial rise of the magnetic flux tubes and their first contact (Sect. 3.1). Second, we discuss the formation of the current sheet, the formation of plasmoid-like structures and 


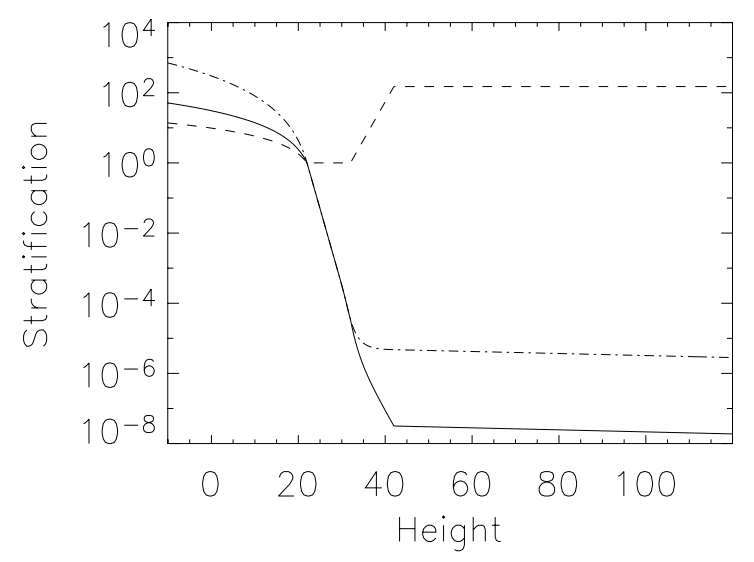

Fig. 1. Distribution of temperature (dashed line), pressure (dot-dashed) and density (solid) along height $(x=0)$ at $t=0$.

the dependence of the reconnection rate on the resistivity model (Sect. 3.2). Then, we study the changes of the fieldline connectivity due to reconnection, the formation of arcade-like flares and loop brightnenings and the time evolution of the current sheet (Sects. 3.3 and 3.4). Finally, Sect. 4 contains a summary of conclusions from the results of the numerical experiments.

\section{The numerical model}

The code used to simulate the evolution of the model is a $2.5 \mathrm{D}$ version of the Lagrangian remap scheme detailed in Arber et al. (2001) that has been used by Leake \& Arber (2006) to study the effect of partial ionisation and Newton cooling in flux emergence experiments. The evolution of the system is governed by the following time-dependent and resistive MHD equations (in a dimensionless form).

$$
\begin{aligned}
& \frac{\partial \rho}{\partial t}+\nabla \cdot(\rho \boldsymbol{u})=0, \\
& \frac{\partial(\rho \boldsymbol{u})}{\partial t}=-\nabla \cdot(\rho \boldsymbol{u} \boldsymbol{u})+(\nabla \times \boldsymbol{B}) \times \boldsymbol{B}-\nabla \boldsymbol{P}+\rho \boldsymbol{g}, \\
& \frac{\partial(\rho \epsilon)}{\partial t}=-\nabla \cdot(\rho \epsilon \boldsymbol{u})-\boldsymbol{P} \nabla \cdot \boldsymbol{u}+Q_{\mathrm{Joule}}+Q_{\mathrm{visc}}, \\
& \frac{\partial \boldsymbol{B}}{\partial t}=\nabla \times(\boldsymbol{u} \times \boldsymbol{B})+\eta \nabla^{2} \boldsymbol{B},
\end{aligned}
$$

with specific energy density

$$
\epsilon=\frac{P}{(\gamma-1) \rho} \text {. }
$$

The basic quantities used in the simulation are the density $\rho$, the pressure $P$, the magnetic field vector $\boldsymbol{B}$ and the velocity vector $\boldsymbol{u}$. $Q_{\text {Joule }}$ is the Joule heating term due to resistive losses and $Q_{\text {visc }}$ is the viscous dissipation, $\boldsymbol{g}$ is the gravity and is taken to be uniform in the $y$-direction, $\eta$ is the resistivity and $\gamma$ is the ratio of specific heats. We used the above code to perform experiments with uniform resistivity and with locally enhanced resistivity (so that the resistivity is switched on only when the current has exceeded some critical value). The code also includes small shock viscosity and viscous heating.

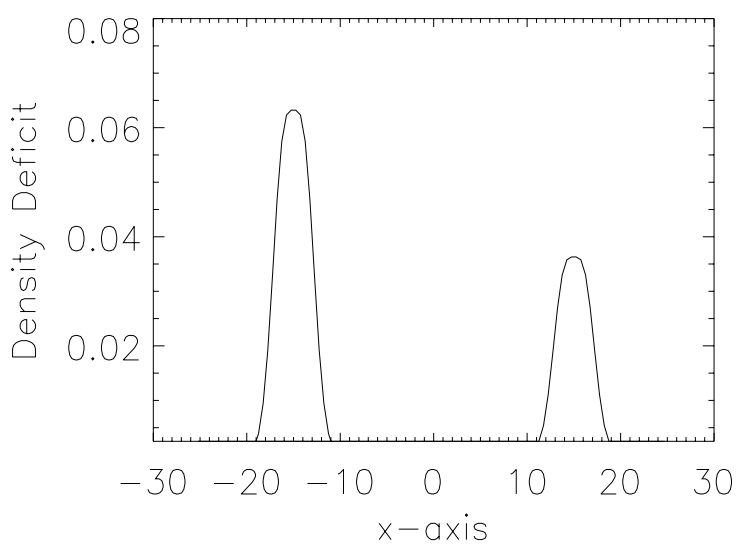

Fig. 2. Horizontal distribution of $|\Delta \rho| / \rho$ for the two flux tubes at heights $y=8.8$ (left tube) and $y=4.8$ (right tube) at $t=0$.

The initial stratification of the atmosphere is similar to the model used in Archontis et al. (2005). Figure 1 shows the distribution of temperature, pressure and density with height at the center of the computational volume. All variables are made dimensionless against their photospheric values. The photospheric values are: pressure, $p_{\mathrm{ph}}=1.4 \times 10^{5} \mathrm{erg} \mathrm{cm}^{-3}$; density, $\rho_{\mathrm{ph}}=$ $3 \times 10^{-7} \mathrm{~g} \mathrm{~cm}^{-3}$; temperature, $T_{\mathrm{ph}}=5.6 \times 10^{3} \mathrm{~K}$ and scale height, $H_{\mathrm{ph}}=170 \mathrm{~km}$. Other units used in the simulations are: time, $t_{\mathrm{ph}}=25 \mathrm{~s}$; velocity, $V \equiv\left(p_{\mathrm{ph}} / \rho_{\mathrm{ph}}\right)^{1 / 2}=6.8 \mathrm{~km} \mathrm{~s}^{-1}$ and magnetic field, $B_{\mathrm{ph}}=1.3 \times 10^{3}$ Gauss.

The size of the grid used in the numerical experiments is $(400,800)$ points in the $(x, y)$ directions, with $y$ being the vertical coordinate. The dimensionless coordinates are $(-100 \leq x \leq$ $100)$ and $(-10 \leq y \leq 120)$. The stratified atmosphere includes the top of the solar interior from $y=-10$ to 22 , two isothermal layers $(y=22$ to 32 for the photosphere and $y=42$ to 120 for the corona) and a transition region with a steep temperature gradient joining them. The grid has a uniform spacing corresponding to $0.027 \mathrm{Mm}$ and $0.083 \mathrm{Mm}$ in the $y$ and $x$-directions. Both of these scales are everywhere smaller than the relevant scale heights. Damping regions are implemented on the top and side boundaries.

At $t=0$ two magnetic flux tubes are located below the photosphere, at $y=8.8$ and $y=4.8$ respectively, having their axes perpendicular to the $x y$ plane. The initial horizontal distance between the axes of the tubes is $30(\approx 5 \mathrm{Mm})$ and their vertical distance is $4(\approx 0.7 \mathrm{Mm})$. The value of the radius of the tubes is $R=2.5$. The magnetic field on the axis has a $B_{z}$ component, which is given by a simple Gaussian profile

$B_{z}=B_{0} \exp \left(-\frac{r^{2}}{R^{2}}\right)$

where $r=\sqrt{x^{2}+\left(y-y_{0}\right)^{2}}$ is the radial distance from the axis of the tube and $y_{0}$ is the initial depth of each tube. The magnetic field strength on the axes of the tubes is $B_{0}=5$. This field strength corresponds to a local plasma $\beta=13.4$ and 7.5 at the axis of the left and the right tube respectively, with $\beta$ being the ratio of the gas pressure to the magnetic pressure.

The magnetic field lines are uniformly twisted around the central axes of the tubes, $B_{\varphi}=\alpha r B_{z}$, with $B_{\varphi}$ the azimuthal component of the magnetic field in the $x y$ plane and $\alpha=0.4$. With this twist, the tubes are marginally unstable to the kink instability. Additionally, both tubes have a left-hand twist and, 
thus, the fieldlines on the right side of the first tube are oppositely directed to the fieldlines on the left side of the second tube.

To initiate the experiment, a density deficit is introduced into the tubes (Archontis et al. 2004). It has maximum at the axis of each tube and generates the classical Parker buoyancy instability. Figure 2 shows $|\Delta \rho| / \rho$ for the two tubes. $\Delta \rho$ is the absolute value of the density excess and $\rho$ is the density of the background atmosphere. $\Delta \rho$ is measured across the center of the tubes along the $x$-axis and $\rho$ takes the value of the non-magnetic atmosphere at the heights of the tube's centers. Due to the density deficit, the central part of each tube is underdense and the buoyancy force causes the two flux tubes to rise. Notice, that the value of $|\Delta \rho| / \rho$ is higher for the left tube because it is originally located at a higher level in the subphotospheric layer compared to the right tube.

\section{Results}

\subsection{Initial rise and contact}

Figure 3 shows the rise until contact of the two flux tubes. The top of the left tube reaches the photosphere at $t=37$ $(\approx 15 \mathrm{~min})$ and moves $\approx 1 \mathrm{Mm}$ inside the photosphere by $t=57$, while the right tube remains inside the subphotospheric layer (Fig. 3, second panel). The leading tube starts to expand because the magnetic pressure is larger than the gas pressure outside the magnetized volume. The shape of the tubes is slightly deformed because the gas pressure in the region between the tubes $(8<x<20$ and $-9<y<9)$ is slightly enhanced. The leading (following) tube expands more towards the left (right) boundary of the box.

At $t=75$ (Fig. 3, third panel), the leading tube is now well inside the corona and expands, due to excess magnetic pressure, to create a fan-like, ambient coronal field. Figure 4 is a closeup of the expanding magnetic field of the first tube at $t=86$. The image shows the intensity of the magnetic field indicating that $\boldsymbol{B}$ decreases away from the central axis of the tube, which is located at $x=-15, y=20$. The arrows are the projection of the magnetic field vector on the $x y$ plane and show the direction of the fieldlines. The figure shows that the ambient field is directed downward (and almost antiparallel to the $y$-axis at $x=15$, $y=43$ ) and eventually changes direction and becomes antiparallel to the direction of the uppermost fieldlines of the upcoming second flux tube. Thus, the ambient field in this experiment is non-uniform (in strength and direction) and more complex than the ambient fields used in similar previous simulations.

At $t=100$ the outer edge of the second tube is at $y=4.2$ $(\approx 0.7 \mathrm{Mm}$ above photosphere) and comes into contact with the lower part of the fan-like ambient field of the leading tube (Fig. 3, bottom panel), forming a strong current sheet, whose evolution is discussed below.

\subsection{Current sheet and plasmoid-like structures}

A thin current sheet is formed at the interface of the two flux systems as they first come into contact. The sheet takes the form of an arch as the field of the second tube rises and pushes the ambient field upward. Figure 5 shows the time evolution of the axial component of the current density $J_{z}$ and the full magnetic field vector (arrows). At $t=120$ (top panel) the upper part of the arch is at $y=56$ (a height of $2.4 \mathrm{Mm}$ inside corona) while the footpoints of the current arch remain in the photosphere with an horizontal separation of $x=20(\approx 3.4 \mathrm{Mm})$.
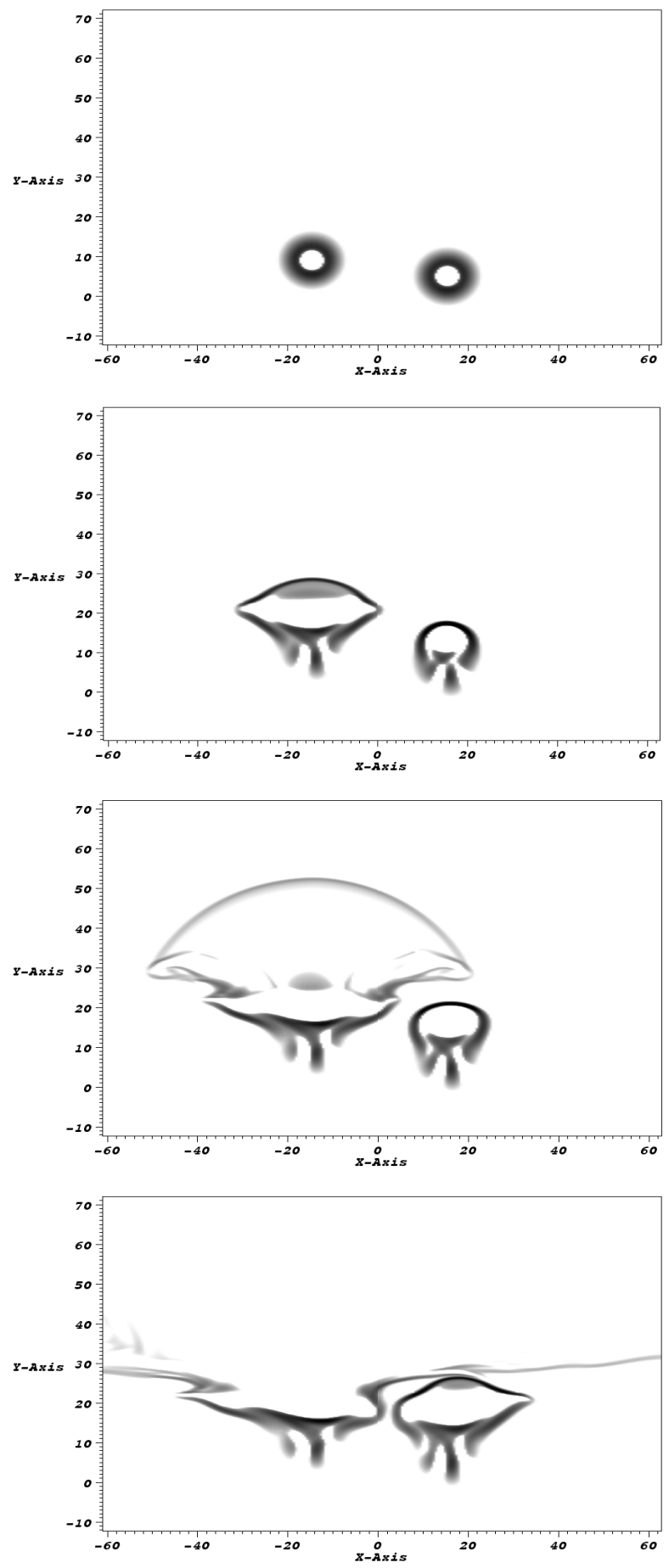

Fig. 3. Time evolution of the pair of the tubes. The panels show the intensity in $J_{z}$. Times are $t=0,57,75$ and $t=100$ for the top, middle and bottom panels correspondingly.

During the evolution, there is a difference between the magnetic pressure above and below the current sheet, resulting in a non uniform expansion of the magnetized volume below the current sheet into the atmosphere. Expansion is faster towards the right side of the domain where the ambient field is weaker (Fig. 5, top panel). Also, the magnitude of the current density and the thickness of the interface are larger towards the left side of the current arch, in the region between the two expanding magnetic tubes and near the center of the computational domain, than on the right side. 


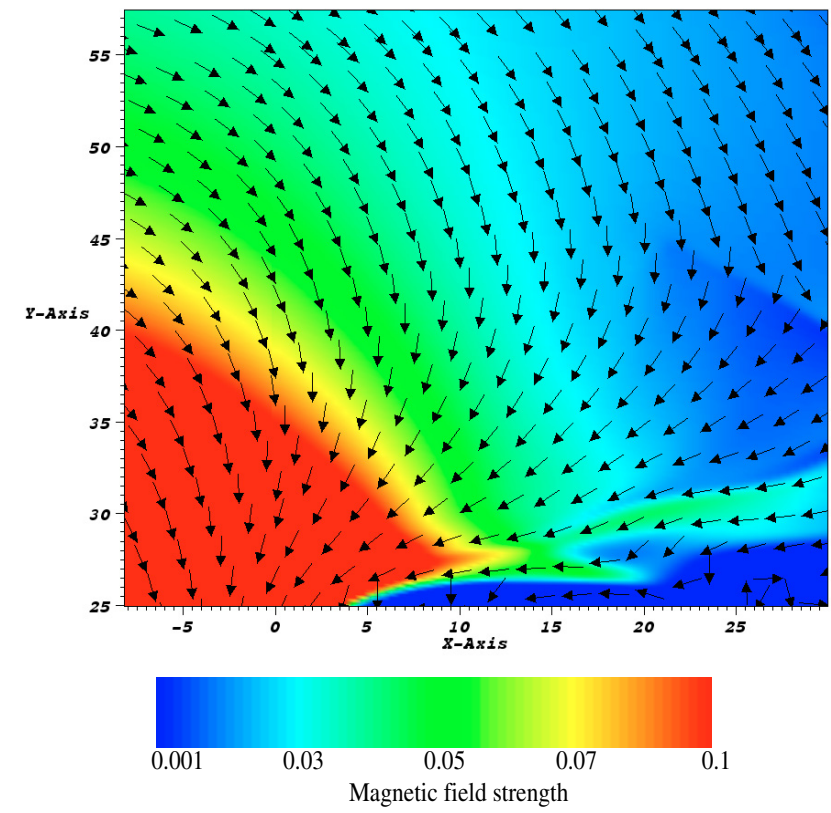

Fig. 4. Close-up of the expanding magnetized volume of the first tube at $t=86$. The image is a visualization of the strength of the magnetic field. The arrows show the direction of the full magnetic field vector.

The top panel in Fig. 5 shows the direction of the magnetic field on either side of the discontinuity and reveals that reconnection of fieldlines occurs mainly close to the left and right sides of the current arch. The contact of the two flux systems along the current arch is restricted because magnetic islands (or plasmoids) are formed inside the current sheet. At $t=136$ the distance between the islands is $\approx 9.5$ times the thickness of the current sheet. This is comparable with the most unstable wavelength of the tearing mode instability (e.g., Priest 1982).

The middle panel in Fig. 5 shows the formation of two plasmoids within the current sheet at $(x, y)=(7,53)$ and $(x, y)=$ $(14,63)$ at $t=140$. They contain cool, dense chromospheric plasma with enhanced current density. The upper plasmoid, close to the top of the current sheet, is expelled out of the arch, due to the magnetic tension of the reconnected fieldlines. After the plasmoid's ejection, the current arch splits into two separate current sheets. One sheet extends from $(x, y)=(14,69)$ to $(x, y)=(45,31)$ and it is connected with the ejected plasmoid on its left end. The other current sheet is located at the interface between $(x, y)=(2.5,40)$ and $(x, y)=(11,65)$ and contains the lower plasmoid, which is expelled from the sheet after $t=140$.

The size of the plasmoids grows exponentially in time indicating the occurrence of a linear instability (such as the tearingmode instability) in the current sheet. Figure 6 shows the temporal evolution of the width of the plasmoid which is formed at the lower left flank of the current sheet at $t \approx 136$. Initially, the plasmoid grows exponentially until the time at which is ejected out of the current sheet. After the ejection $(t \approx 140)$, the size of the magnetic island does not change dramatically. The formation and evolution of plasmoids in 2D and 3D geometry has also been studied in previous simulations (Yokoyama \& Shibata 1995; Magara et al. 1997; Archontis et al. 2006).

After the ejection of the plasmoid (bottom panel in Fig. 5), two pairs of slow shocks (with V-shaped topology) are formed at the ends of the vertical current sheet. Figure 7 shows the distribution of the perpendicular component of the current at $t=152$. The thin vertical stripe in the middle of the figure is the current sheet. The pairs of the slow shocks start from the ends of the
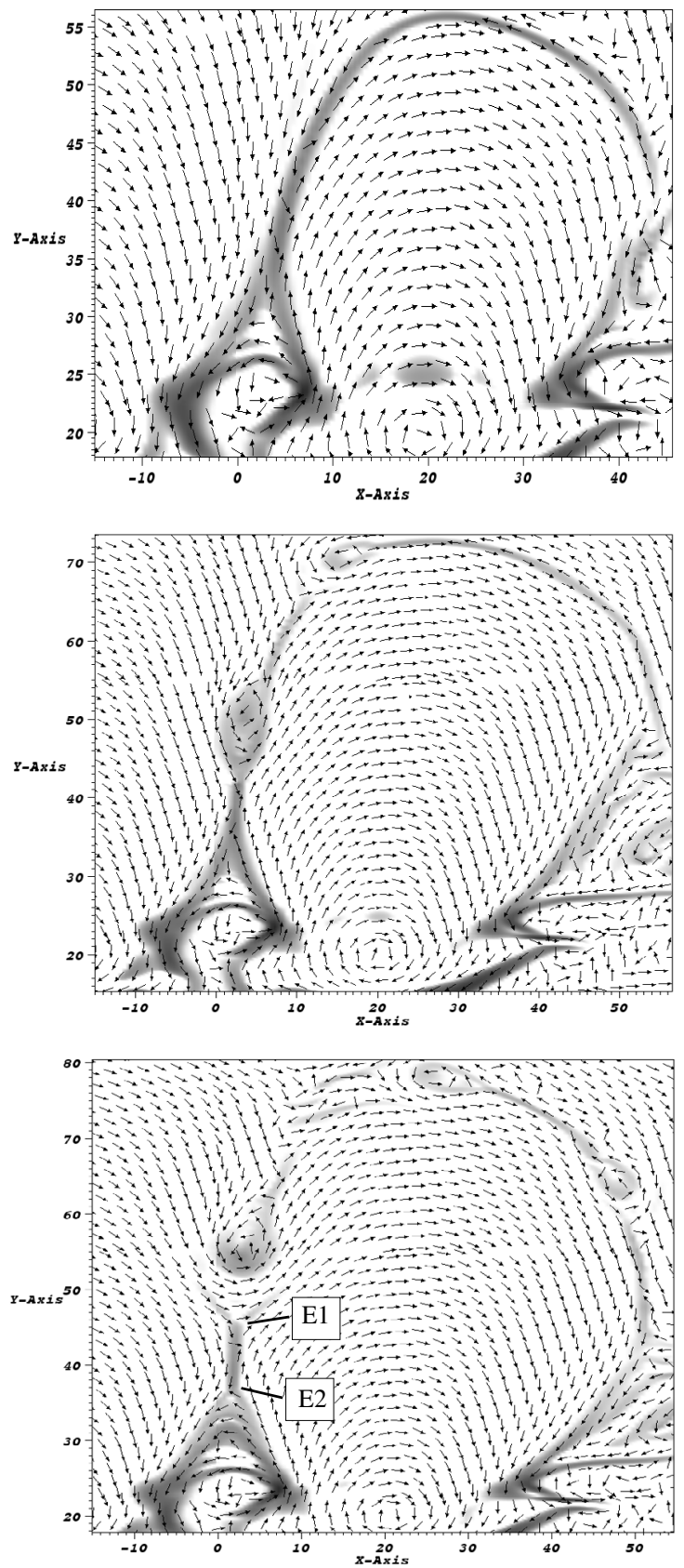

Fig. 5. Time evolution of the current arch and the plasmoids. The grayscale image represents the intensity in $J_{z}$ and the arrows the full magnetic field vector. Times are $t=130,140$ and $t=145$ for the bottom, middle and bottom panel correspondingly. E1 and E2 label the ends of the vertical current sheet. The resistivity for this experiment is $\eta=0.001$.

current sheet at $y=39$ and $y=52$. The arrows show the full velocity field vector. At the center of the current sheet, there is a relative horizontal inflow because of the sideway expansion of the two magnetic flux systems. At the ends of the current sheet, the plasma is ejected upward and downward because of reconnection of magnetic fieldlines of the two magnetic flux systems. The velocity flow changes direction noticeably at the sites of the slow shocks. Figure 8 shows the horizontal distribution of the total electric current (top panel), the magnetic and gas pressure (middle panel) and the density (bottom panel) along $y=38$ at $t=152$. The current density is large at the sites of the slow shocks. The magnetic pressure decreases as the shock passes 


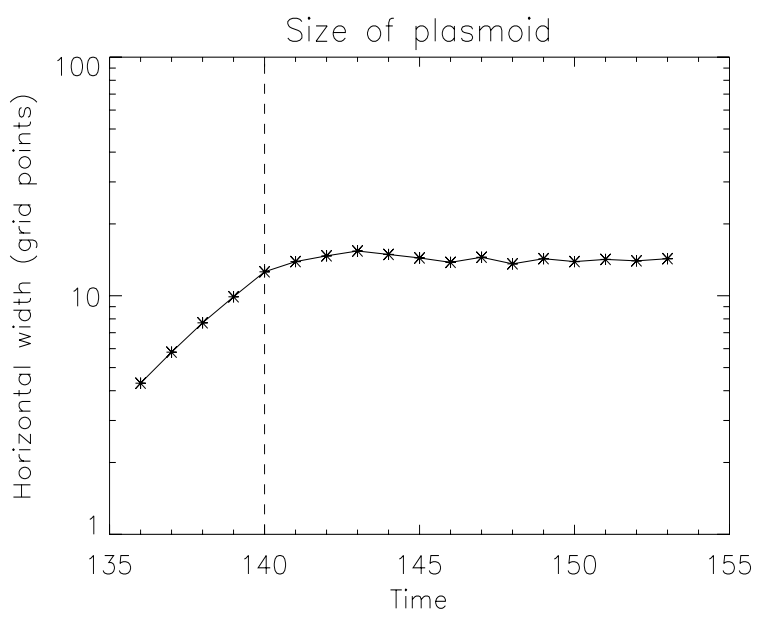

Fig. 6. Temporal evolution of the size of the plasmoid.

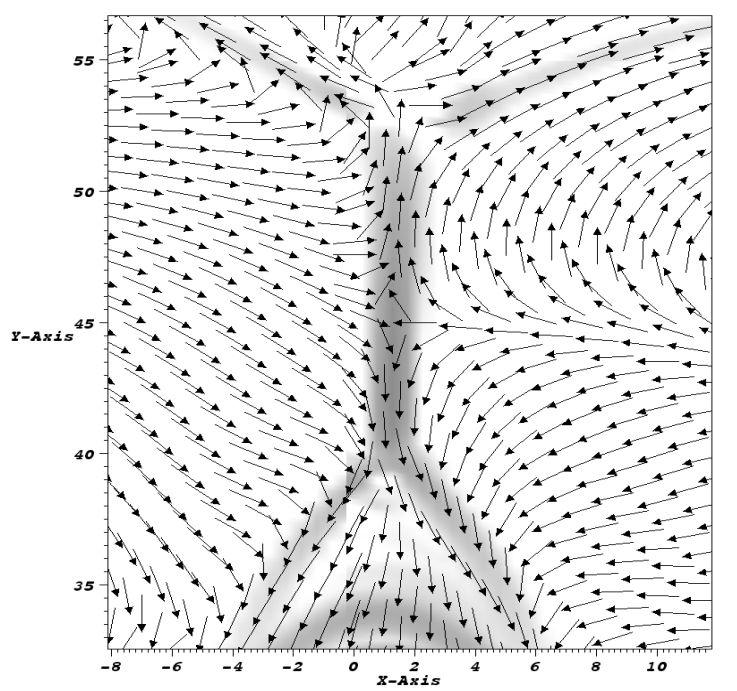

Fig. 7. Distribution of $J_{z}$ around the vertical current sheet at $t=152$. Arrows show the velocity field vector.

while the gas pressure increases ahead the shock, the result being a change in the total pressure across the shock. The density increases at the site of the left slow shock. The decrease of density at the site of the right shock is because, at this time of the simulation, hot plasma is ejected from the reconnection region and is accelerated along this region at the site of the slow shock.

The existence of slow-mode MHD shocks, extending from the current sheet, is characteristic of Petschek-type reconnection. We calculate the reconnection rate at the vertical current sheet starting at $t=137$, just before the ejection of the plasmoid from the sheet. The reconnection rate is defined as

$\epsilon=\frac{v_{\text {in }}}{v_{\text {alf }}}$,

where $v_{\text {in }}$ is the inflow velocity toward the diffusion region and $v_{\text {alf }}$ is the Alfven velocity. The inflow velocity is calculated as the maximum absolute value of the difference

$v_{\text {in }}=v_{x}\left(x_{a}, L_{y}\right)-v_{x}\left(x_{b}, L_{y}\right)$,

where $v_{x}$ is the horizontal component of the velocity, $x_{a}$ and $x_{b}$ are points (along the $x$-axis) located away from the diffusion
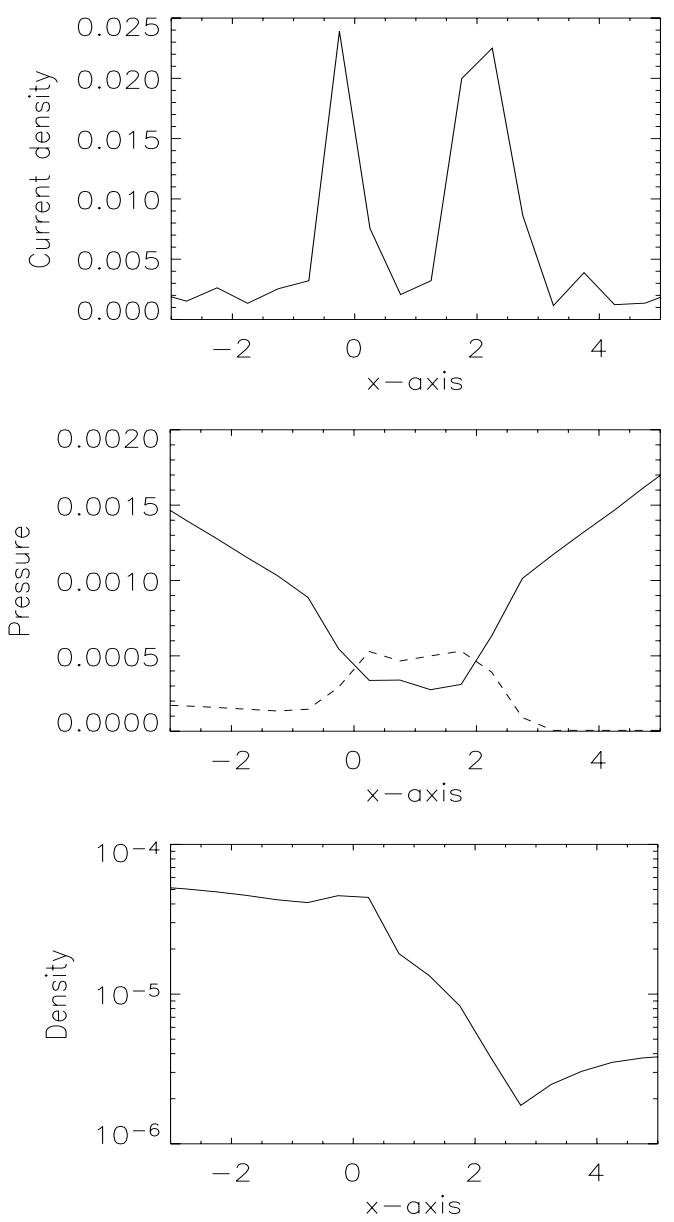

Fig. 8. Top panel: distribution of current density. Middle panel: distribution of magnetic pressure (solid line) and gas pressure (dashed line). Bottom panel: distribution of density.

region and $L_{y}$ is the vertical size of the current sheet. The Alfven velocity is defined as

$v_{\mathrm{alf}}=\sqrt{\frac{B^{2}}{2 \rho}}$,

where $B$ is the magnetic field strength and $\rho$ is the density. The Alfven velocity is calculated, similar to the inflow velocity, away from the diffusion region and at the point along $L_{y}$ where maximum inflow velocity is attained.

Figure 9 shows the time evolution of the reconnection rate. At the beginning the rate is small and increases rapidly after the ejection $(t \approx 140)$ of the big plasmoid from the current sheet. The increase is mainly due to the large inflow velocity toward the diffusion region after the upward movement of the plasmoid along the current sheet. The maximum value obtained is $\epsilon \approx 0.11$ at $t=144$. This large value of the reconnection rate indicates that reconnection is likely to be fast. The second peak of the reconnection rate at $t=148$ is due to a secondary ejection of a smaller plasmoid out of the current sheet. After the ejection of the magnetic islands the current sheet collapses and the reconnection rate takes on smaller values close to $\epsilon \approx 0.03$.

We performed two more experiments to investigate the effect of the resistivity on the formation of the plasmoids and the reconnection rate.

In the first experiment the value of the uniform resistivity is much larger, $\eta=0.02$. Figure 10 shows the time evolution of 


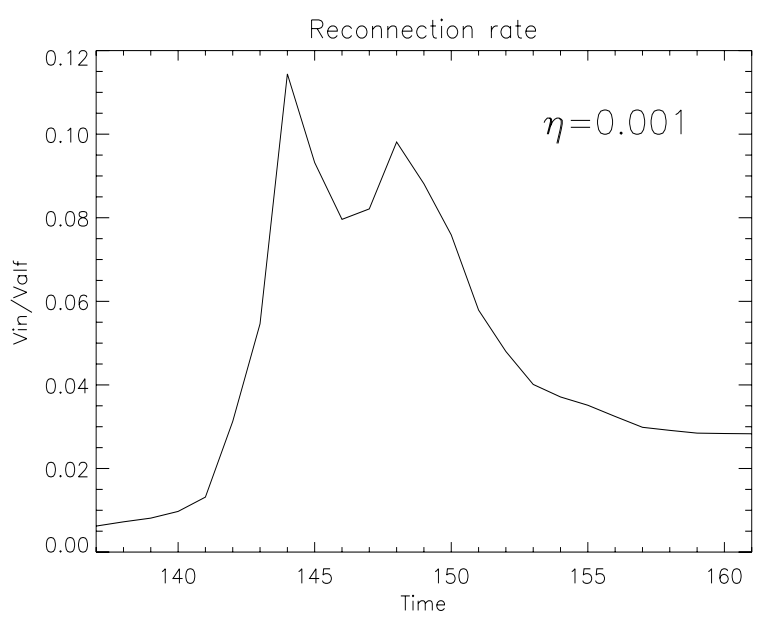

Fig. 9. Time evolution of the reconnection rate around the vertical current sheet for the uniform resistivity case with $\eta=0.001$.

the current arch, which is formed between the upcoming flux system, and the ambient field, which is formed by the expansion of the first rising flux tube (and should be compared with Fig. 5 where $\eta=0.001$ ). The first panel shows that there is a plasmoid at $x=26$ and $y=48$, which is moving along the current sheet toward the right-side boundary. Eventually, it is ejected out of the current sheet (middle panel), splitting the arch into two parts. At the lower part a new plasmoid is formed (at $x \approx 13$ and $y \approx 40$ ), which is growing in size (bottom panel) and is expelled from the sheet at a later time. Thus, the general evolution of the system is similar to the $\eta=0.001$ case, although the diffusion is higher and the size of the plasmoid different.

In the second experiment, the resistivity is locally enhanced when the current density grows above a certain value. More precisely,

$\eta= \begin{cases}0.001 & \text { if }|\boldsymbol{J}| \geq 0.01 \\ 0 & \text { if }|\boldsymbol{J}|<0.01 .\end{cases}$

The three panels of Fig. 11 show again the time evolution of the arch-like current sheet and the plasmoids, which are formed inside the diffusion region. The general topology of the current structure and the magnetic islands is very similar to the cases with uniform resistivity, especially with the uniform $\eta=0.001$ case. Finally, we should mention that Archontis et al. (2006) studied the formation of plasmoids inside a curved $3 \mathrm{D}$ current sheet between two magnetic flux systems in a flux emergence experiment. They used a different numerical scheme from the present simulations, with hyper-diffusion coefficients for the numerical resistivity. Thus, it seems that the formation of plasmoids is quite a robust result and not strongly dependent on the resistivity model once there is enough resolution to resolve small scale structures in the experiment.

Figure 12 shows the reconnection rate for the above two experiments, calculated in the same way as for the first experiment with uniform $\eta=0.001$. The top panel corresponds to the case with non uniform resistivity and shows that the time evolution of the reconnection rate is very similar to the case with uniform $\eta=0.001$. In fact, it indicates that there is a parallel evolution of similar structures to the $\eta=0.001$ case, such as plasmoids. The bottom panel corresponds to the case with uniform $\eta=0.02$. The maximum value of the reconnection rate in this experiment is $\approx 0.01$, which is lower than the other two experiments but high enough to indicate that reconnection occurs again with
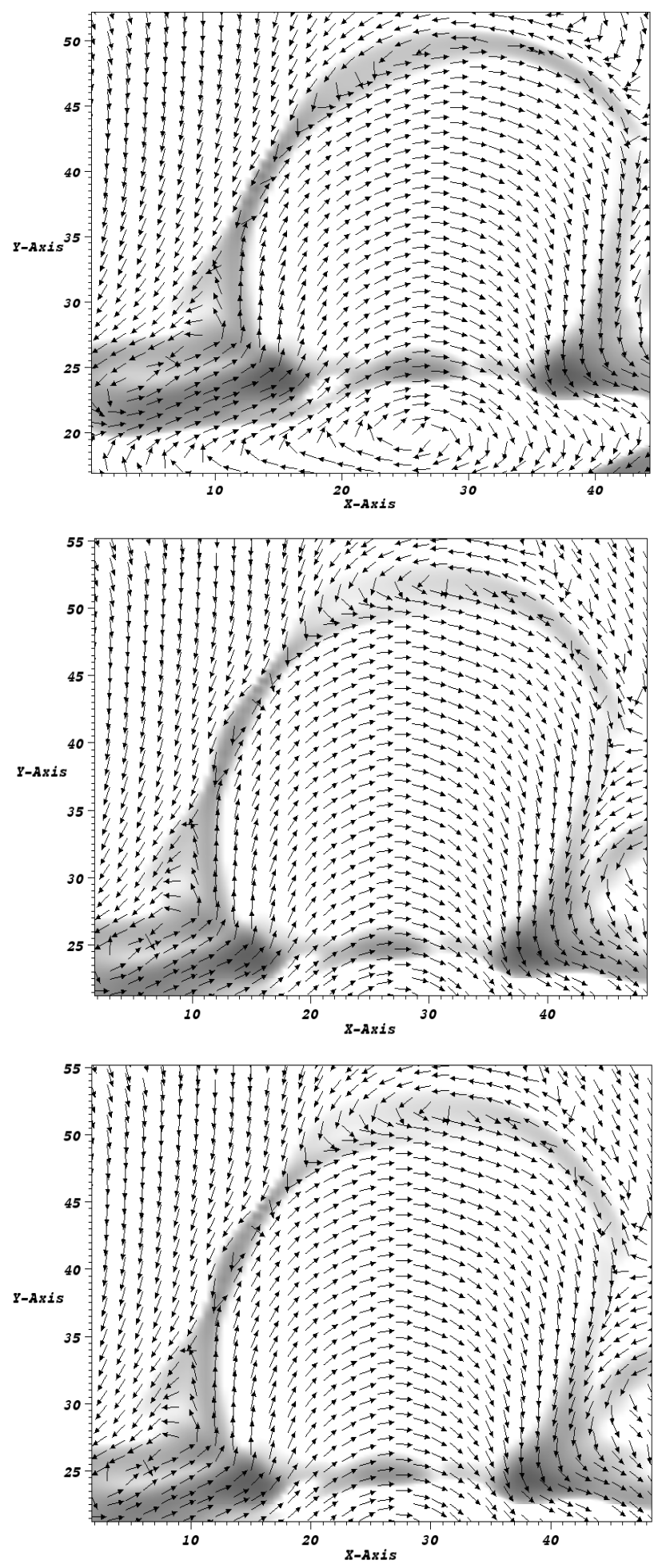

Fig. 10. Time evolution of the current arch and the plasmoids for the case with uniform resistivity, $\eta=0.02$. The gray-scale background represents the intensity of the vertical component of the electric current, $J_{z}$ and the arrows the full magnetic field vector. Times are $t=154,158$ and $t=161$ for the top, middle and bottom panels correspondingly.

a fast rate. The secondary local minima at $t \approx 171$ and $t \approx 177$ correspond to plasmoid ejections of the current sheet. There is a time delay for the occurrence of the first maximum in the reconnection rate compared to the other experiments. This is due to the larger value of the uniform resistivity that affects also the magnetic field strength of the rising magnetic flux systems, their 

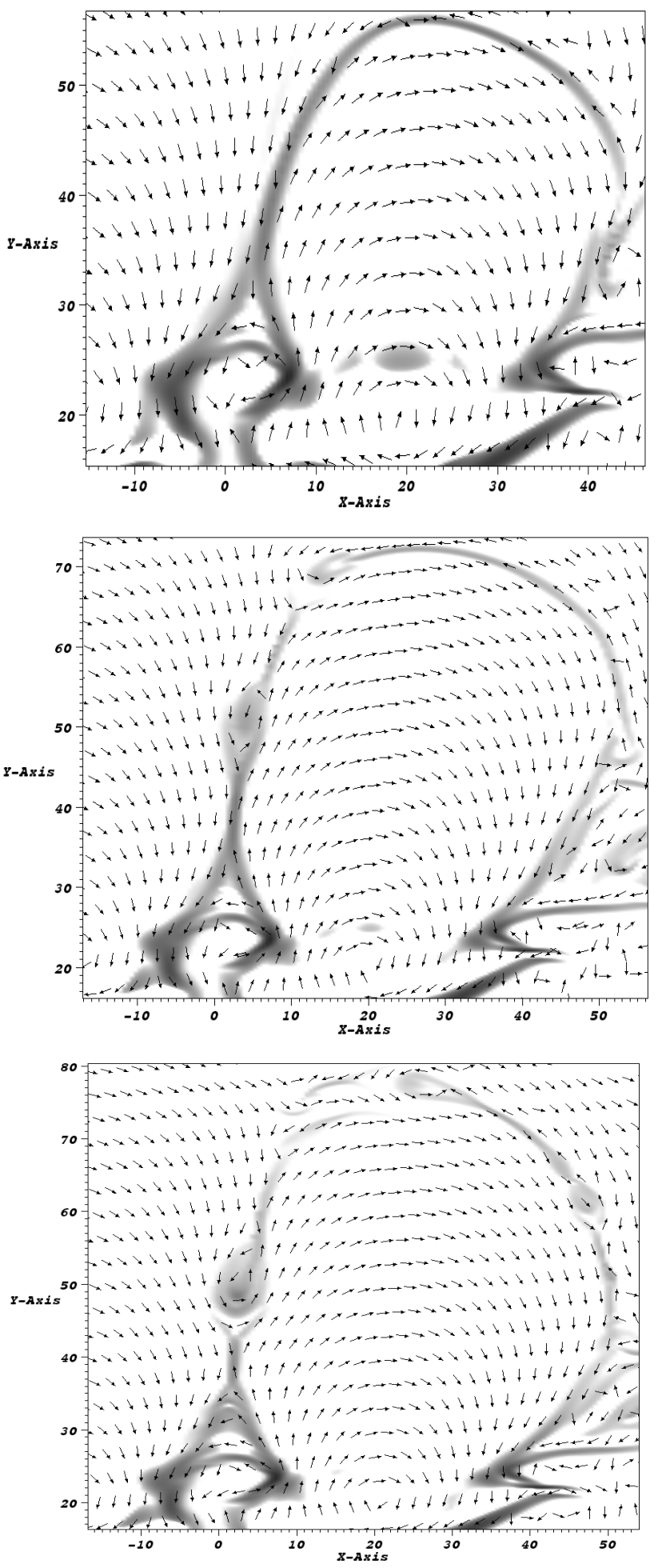

Fig. 11. Time evolution of the current arch and the plasmoids for the case with non uniform resistivity. The gray-scale background represents the intensity of the vertical component of the electric current, $J_{z}$ and the arrows the full magnetic field vector. Times are $t=130,140$ and $t=145$ for the top, middle and bottom panels correspondingly.

buoyancy and, thus, the time-scale of the general evolution of the system.

It is worthwile mentioning that fast reconnection mechanisms and their relation with magnetic islands, formed in a current sheet system, have also been studied by other authors. Ugai (1992) found that the reconnection process strongly depends on
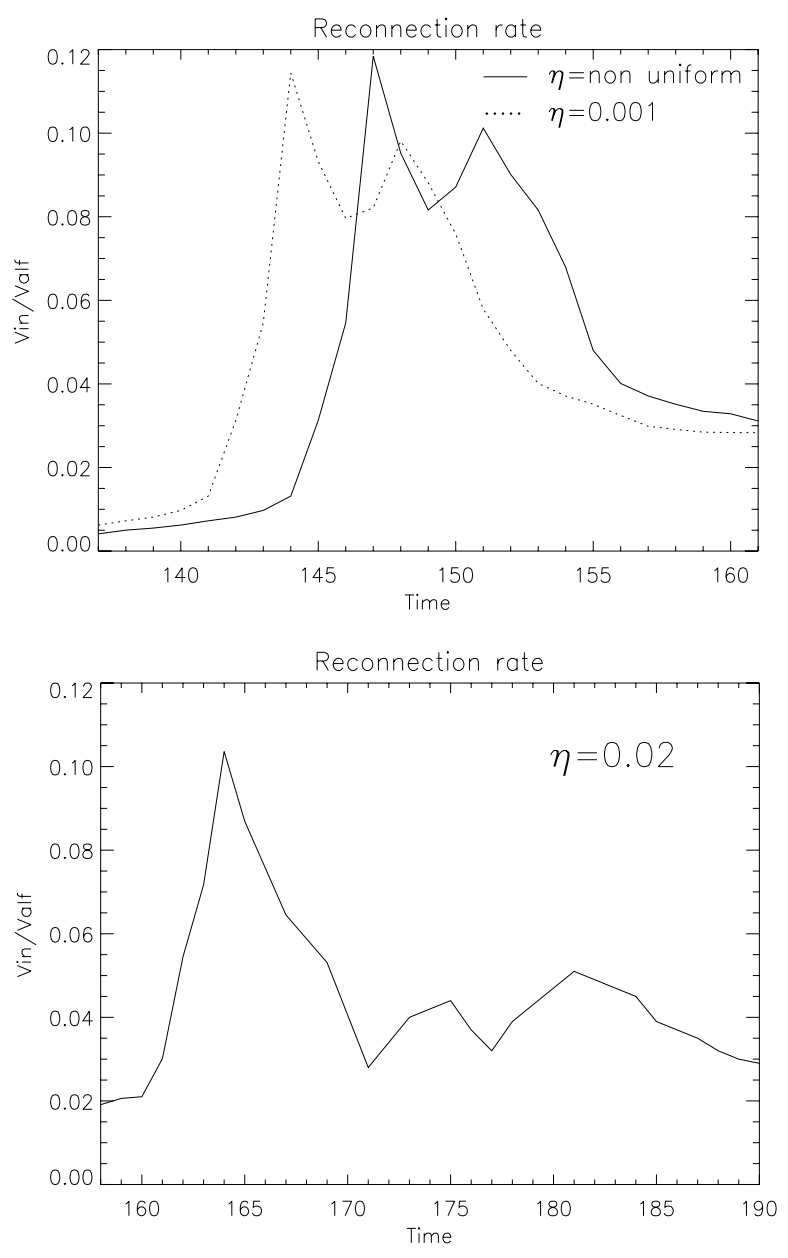

Fig. 12. Top: time evolution of the reconnection rate for the cases with non uniform resistivity (solid line) and with $\eta=0.001$ (dotted line). Bottom: the same for the case with uniform resistivity, $\eta=0.02$.

the resistivity model and fast reconnection occurs only when the resistivity is locally enhanced near an X-type neutral point. Also, Yokoyama \& Shibata (1995) performed 2D MHD simulations of an emerging tube that interacts with an ambient field using uniform and anomalous resistivity. They found that the uniform resistivity case becomes steady Sweet-Parker type, while the anomalous resistivity case becomes a Petschek type with a fast reconnection rate. The above models used a Lax-Wendroff scheme for the numerical computations and large values of resistivity. On the other hand, in our experiments we used a highorder Lagrangian remap shock-capturing code and lower values for the resistivity $\eta$. Our results showed that locally enhanced resistivity is not necessary condition for the occurrence of fast reconnection. Also, an increase of the uniform numerical resistivity by one order of magnitude (from $\eta=0.001$ to $\eta=$ 0.02 ) shows that the maximum reconnection rate decreases only slightly, indicating that the reconnection process remain fast.

\subsection{Fieldline connectivity, jets and loop brightnenings}

As reconnection proceeds and the plasmoids are ejected from the corona, the fieldline topology around the current sheet changes noticeably. Eventually, the fieldlines belong to four different sets according to their connectivity. Two sets of fieldlines belong to the two initial flux systems and have not reconnected yet (regions A and B in Fig. 13). A set of reconnected fieldlines overly 


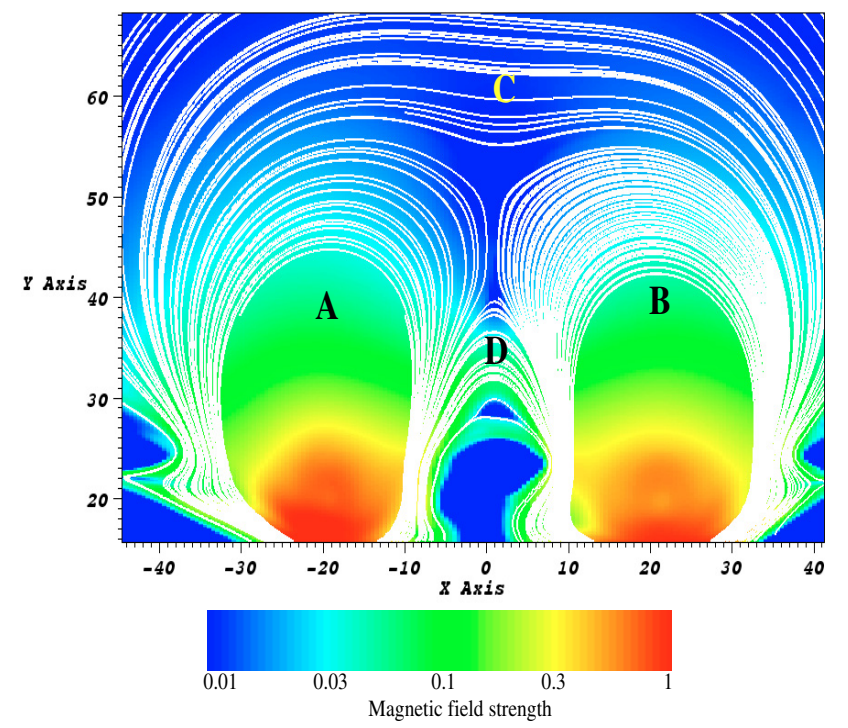

Fig. 13. The magnetic field strength (colormap) and fieldlines (white lines) at $t=154$. The symbols $A-D$ identify regions of distinct magnetic topology.

both regions $\mathrm{A}$ and $\mathrm{B}$ and belong to region $\mathrm{C}$. The fourth set consists of fieldlines which undergo reconnection close to the lower tip of the current sheet (region D). The later set of fieldlines, below the rising flux systems in regions $\mathrm{A}$ and $\mathrm{B}$, form an arcade-like structure. At later times, more of these reconnected fieldlines accumulate at the top of the arcade structure, which expands in size. Also, the temperature of the arcade loop increases.

A similar magnetic field configuration, with that in Fig. 13, has been used by Hirose et al. (2001) as an initial condition for studying arcade flares and dark filaments eruptions. In our experiment this configuration is formed in a self-consistent way. Also, the interaction between the flux systems in regions A and B occurs by the emergence and expansion and not by imposing converging photospheric footpoint motions. Thus, the arcade structure is formed locally by the reconnection process when the two flux systems come close together. Finally, in many other similar numerical experiments a filament is confined initially in a thin current sheet in a quadruple magnetic source model (or a detached flux rope as an ideal candidate for the pre-CME structure) to prevent the release of the stored magnetic energy via magnetic energy. In our simulations, the release of the magnetic energy and the fast reconnection process is triggered by the formation and ejection of plasmoids, which are created inside the current sheet by the tearing-mode instability.

Now, the top panel in Fig. 14 shows high-velocity outflows (arrows) close to the interface between regions A and B. These outflows are jets which enamate from both ends of the current sheet due to reconnection of the oppositely directed fieldlines of regions $\mathrm{A}$ and $\mathrm{B}$.

Below the current sheet, the reconnection outflow is accelerated downward, colliding with the plasma at the top of the magnetic loop in region $\mathrm{D}$. The reconnection of the fieldlines also leads to flux pile-up in the region below the current sheet creating a compression front where the temperature increases up to 2.5 MK. Thus, the general characteristics (widening of the footpoints, increase of temperature) of the arcade structure below the current sheet may account for a compact flare or an arcade flare associated with emerging flux.
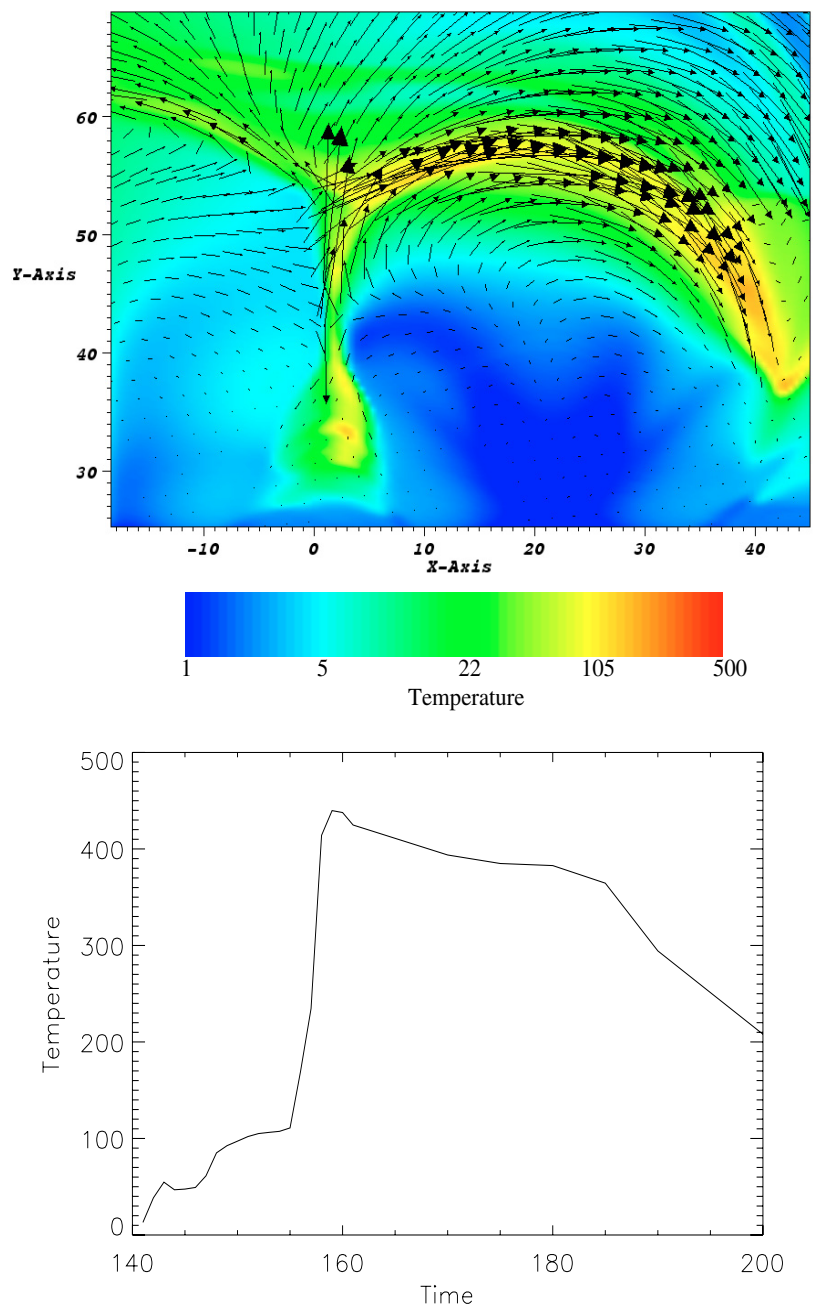

Fig. 14. Top panel: temperature (colormap) and velocity (arrows) around the vertical current sheet at $t=152$. Bottom panel: temporal variation of the maximum temperature of the arcade loop in region D.

Above the current sheet, the reconnection jet collides with the ambient field and the local plasma is compressed. A fast shock is formed there and the plasma is accelerated by the enhanced gas pressure at the compression site along the two seperatrices between regions $\mathrm{C}$ and $\mathrm{A}$ and regions $\mathrm{C}$ and $\mathrm{B}$. Note that the velocity of the accelerated plasma at the right-hand outflow is higher than on the left-hand outflow because the gas pressure increases along the seperatrix between $\mathrm{A}$ and $\mathrm{C}$. The velocity of the accelerated plasma is more than $100 \mathrm{~km} \mathrm{~s}^{-1}$ and the temperature gets as high as $3 \mathrm{MK}$. The temperature enhancement along the upper part of the emerging flux system may account for a loop brightnening.

\subsection{Heating and current sheet collapse}

The bottom panel in Fig. 14 shows the temporal evolution of the maximum temperature in the arcade structure of region $\mathrm{D}$. There are three different phases: first an increase of about one order of magnitude up to time $t=155$, followed by a steep increase (by a factor of 4.3) up to time $t=160$ and a gradual decrease thereafter. The temperature in the first phase is characteristic of the transition region. The sudden and large increase of the temperature between $t=155$ and $t=160$ occurs after the ejection of the last plasmoid from the current sheet. Then, the two flux 

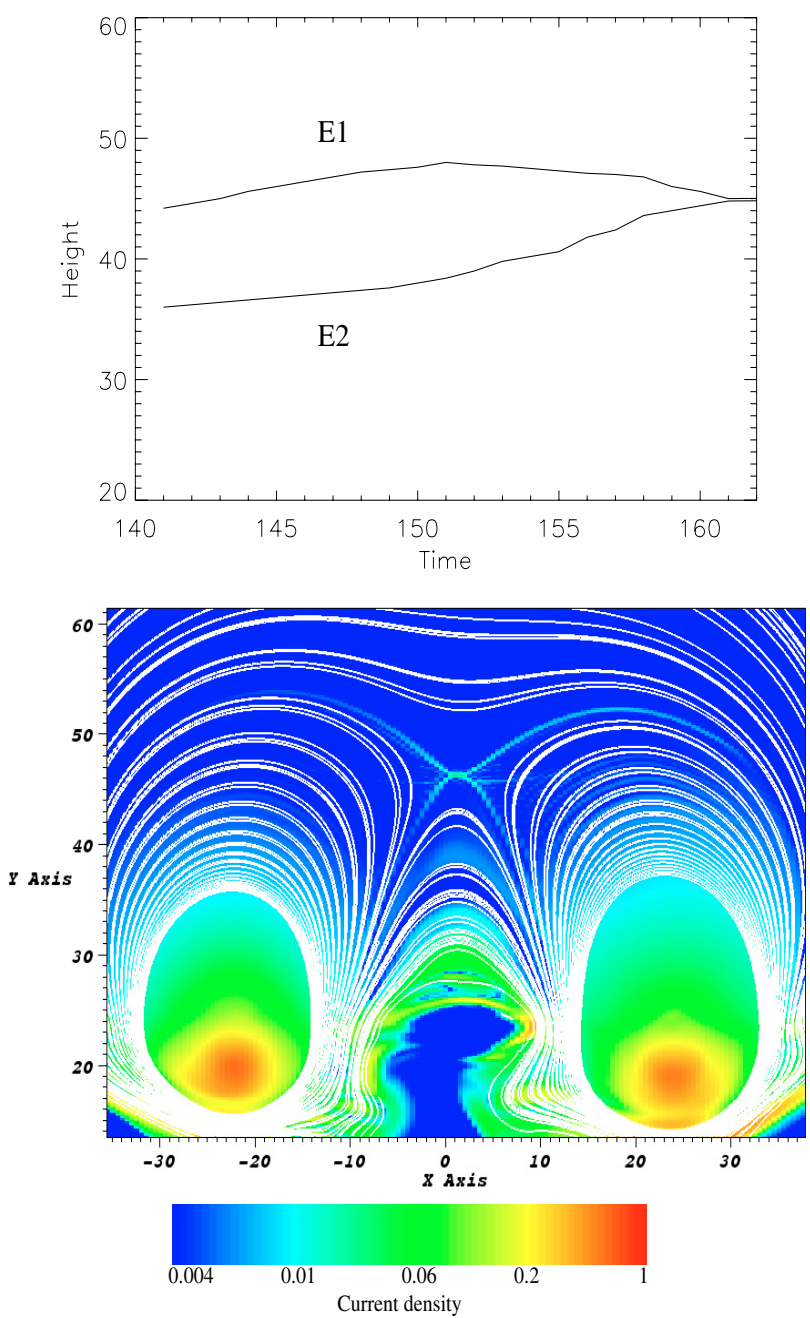

Fig. 15. Top panel: height-time relation of the ends (E1 and E2) of the current sheet after $t=140$. Bottom panel: total current density (colormap) and magnetic field lines at $t=186$.

systems (A and B) come into direct contact, effective reconnection occurs and hot plasma starts to be ejected downward from the diffusion region. At later times (after $t=160$ ), the temperature decreases and gradually return to a value closer to the unperturbed coronal value. The decrease of the temperature is associated with the dynamics of the current sheet and the reconnection process as explained below.

The top panel in Fig. 15 shows the heights of the ends of the vertical current sheet as a function of time. The distance between the solid lines is a measure for the length of the current sheet. There are two prominent phases in the figure. In the first phase (up to $t \approx 152$ ) the current sheet rises keeping an almost constant length. From then on, the current sheet shrinks in length and eventually collapses to an X-point $(t \approx 160)$. We note that the collapse of the current sheet starts shortly after the final plasmoid ejection at $t \approx 148$.

After the ejection of the plasmoids, the continuous reconnection process that occurs in the diffusion region rapidly dissipates the current sheet and the magnetic field approaches a potential state. As a result of this dynamic evolution of the system, the current sheet length collapses to an X-type point, with four branches where the current density is relatively enhanced (bottom panel in Fig. 15 around location $(0,47)$ ). A similar field configuration was found in Mackay \& van Ballegooijen (2006a).
The four branches, which meet at the X-type point, act as seperatrices between the flux regions A, B, C and D. The flow topology around the $\mathrm{X}$-type point is now such that there is a relative inflow along the $y$-axis and the (weak) outflows are emitted horizontally. Thus, the emission of hot plasma from the current sheet towards the top of the rising arcade-loop in region D stops and consequently the temperature decreases, as shown in the bottom panel in Fig. 14.

\section{Conclusions}

We performed 2.5D MHD simulations to study the interaction between two emerging flux systems, which are initially located in subphotospheric heights. One of the key features is that the first flux system emerges and forms a non uniform coronal field that the second flux system emerges into. The results of the interaction of the two magnetic flux systems show that many dynamical phenomena are produced by this new model in a selfconsistent manner.

A current sheet is formed at the interface between the two interacting flux systems when they come into contact. Intense Ohmic heating occurs inside the diffusion region and plasmoids are formed there due to resistive instabilities. The current sheet is divided in various parts when the plasmoids are ejected from the sheet. The ejection of the cool and dense material of the plasmoids into the outer atmosphere might be comparable to $\mathrm{H} \alpha$ and $\mathrm{H} \beta$ surges. Two pairs of slow shocks are formed at the tips of the current sheet. Fast reconnection occurs and reconnection jets collide with external fields to create fast shocks.

It is important to stress that the entire evolution is due to the emergence of firstly one field, to produce a coronal field, followed by the emergence of a second field. There are no horizontal velocities important at the photospheric level as in other models.

Experiments with uniform and non-uniform resistivity have been performed and showed that the reconnection rate remains high in both cases. Now, the hot compressed plasma in the fastshock region and the hot outflows, emitted sideways from the top and bottom ends of the vertical current sheet, may account for dynamic features observed in the Sun by the Yohkoh and Trace satelites, such as X-ray bright points and X-ray jets correspondingly. Especially, the hot plasma which is ejected along the reconnected fieldlines at the top of the second emerging flux tube may account for a loop brightnening. Also, the reconnected fieldlines form a magnetized loop beneath the lower end of the current sheet. The characteristics of this loop are comparable for a compact flare or an arcade flare associated with emerging flux. Finally, the evolution of the system shows the formation of separatrices between four different magnetic flux domains and the relaxation of the vertical current sheet between the two emerging flux systems to an X-type point.

It is worthwile to mention that heating terms are not included in the code, apart from the dissipation terms in the adiabatic energy equation. However, these heating terms are important for the thermodynamical description of the system. In the corona, for example, thermal conduction is a dominant term in the energy balance. The temperature profiles that we obtain throughout the simulation are likely to be modified when heating sources will be included in the experiments.

Although our model is simple, it relates the magnetic field below the photosphere to the structure and eruptive events of the corona. It suggests that plasmoid, reconnection jets, loop brightnenings and arcade flares are coupled with the emergence 
and interaction of magnetic field structures from within the solar interior. Further work, including 3D simulations, is necessary to understand the energetics and dynamics of the interaction of emerging flux systems.

\section{References}

Arber, T. D., Longbottom, A. W., Gerrard, C. L., \& Milne, A. M. 2001, J. Comp. Phys., 171, 151

Archontis, V., Moreno-Insertis, F., Galsgaard, K., Hood, A., \& O’Shea, E. 2004 A\&A, 426, 1047

Archontis, V., Moreno-Insertis, F., Galsgaard, K., \& Hood, A. 2005, ApJ, 635, 1299

Archontis, V., Moreno-Insertis, F., Galsgaard, K., \& Hood, A. 2006, ApJ, 645, L161

Fan, Y., Zweibel, E. G., \& Lantz, S. R. 1998, ApJ, 493, 480

Galsgaard, K., Moreno-Insertis, F., Archontis, V., \& Hood, A. 2005, ApJ, 618, 153
Heyvaerts, J., Priest, E. R., \& Rust, D. M. 1977, ApJ, 216, 123

Hirose, S., Uchida, Y., Uemura, S., Yamaguchi, T., \& Cable, S. B. 2001, ApJ, 551,586

Isobe, H., Miyagoshi, T., Shibata, K., \& Yokoyama, T. 2005, Nature, 434, 478

Isobe, H., Tripathi, D., \& Archontis, V. 2007, ApJ, 657, L53

Leake, J. K., \& Arber, T. D. 2006, A\&A, 450, 805

Linton, M., Dahlburg, R. B., \& Antiochos, S. K. 2001, ApJ, 553, 905

Mackay, D., \& Ballegooijen, A. A. 2006a, ApJ, 641, 577

Mackay, D., \& Ballegooijen, A. A. 2006b, ApJ, 642, 1193

Magara, T., Shibata, K., \& Yokoyama, T. 1997, ApJ, 487, 437

Murray, M. J., \& Hood, A. W. 2007, A\&A, submitted

Priest, E. R. 1982, Solar Magnetohydrodynamics (Dordrecht: D.Reidel Publishing Company), 206

Shibata, K., Masuda, S., Shimojo, M., et al. 1995, ApJ, 451, 83

Ugai, M. 1992, Phys. Fluids B, 4, 9

Yokoyama, T., \& Shibata, K. 1994, ApJ, 436, 197

Yokoyama, T., \& Shibata, K. 1995, Nature, 375, 42

Yokoyama, T., \& Shibata, K. 1996, Publ. Astron. Soc. Japan, 48, 353 